\title{
Hormones and Cancer: an Exciting Young Journal for an Expanding Field of Basic, Translational, and Clinical Study
}

\author{
Nancy L. Weigel ${ }^{1}$
}

Received: 30 March 2015 / Accepted: 30 March 2015 / Published online: 14 April 2015

(C) Springer Science+Business Media New York 2015

It is an honor to have been selected to serve as the editor-inchief of Hormones and Cancer, the newest of the Endocrine Society journals (published jointly by the Endocrine Society and Springer). I admire and commend the founding editor-inchief, Dr. Jonathan Li, and the second (and immediate past) editor-in-chief, Dr. Carol A. Lange, for their accomplishments in establishing and further developing this relatively new journal. I have served on the Editorial Board since its inception, and it has been a pleasure to see the progress the journal has made from a good idea to a promising journal with increasing submissions and impact. One of the successes during Dr. Lange's tenure was in recruiting experts in androgen receptor splice variants in prostate cancer and experts in thyroid cancer to write two series of excellent and timely reviews on these topics. The journal is now indexed in PubMed and will soon receive its first Impact Factor. My immediate goal is to increase the visibility and impact of the journal in basic, translational, and clinical studies of hormone-dependent cancers and cancers of endocrine organs that produce hormones. This includes appointing editors and editorial board members with established expertise in the areas covered by the journal, modifications of the journal web sites, additional types of journal content, and increased advertising. My ultimate goal is to develop the journal so that it is one of the first choices of authors for submission of studies in these topics. The annual meeting of the Endocrine Society routinely includes these topics as major areas of interest for symposia, oral sessions, and poster sessions, but papers on these topics have not previously had a specific Endocrine Society journal home.

Nancy L. Weigel

nweigel@bcm.edu

1 Baylor College of Medicine, Houston, TX, USA
Hormones and Cancer is a novel multidisciplinary journal that covers all aspects of the intersection of endocrinology and cancer. This includes studies of endocrine neoplasias and hormone action in any cancer. Hormones and Cancer is a unique journal for the Endocrine Society in that its articles range from basic science through translational studies to clinical studies and trials. Thus, it addresses the interests of all three Endocrine Society constituencies, basic scientists, clinician scientists, and clinicians in practice.

This is an exciting, but challenging time for all of our constituencies. The rapid development of new technologies provides exciting new opportunities in all areas of study and encourages studies across diverse fields of expertise. Bioinformatics approaches and available databases facilitate studies that span the gamut from basic research to clinical studies. Investigators can quickly determine whether changes observed in vitro or in pre-clinical models are also observed in clinical specimens.

Scientists and clinicians alike are facing many challenges with reduced support and funding from NIH and other sources. The publishing industry also has undergone many changes and will continue to change as various forms of electronic publishing increase the types of content (for example, videos) that can be produced and journals alter their access options. With the proliferation of journals including many new online journals not associated either with scientific societies or with established scientific publishers, authors have a bewildering variety of choices for publication. Moreover, there is increasing reliance on PubMed and Google and on key words to identify articles of interest rather than through perusal of tables of contents of a subset of journals. There are a number of benefits to publishing in Hormones and Cancer. The journal offers rapid peer review by experts in the field with clear critiques defining what is needed for publication. Because this is a combined Springer/Endocrine Society 
venture, there are no page charges and the journal is directly accessible online to all 18,000 Endocrine Society members as well as through libraries and individual subscriptions.

To accomplish our goals, several changes have been made with additional changes planned. Two new associate editors have been chosen to ensure adequate coverage of the broad areas of interest covered by the journal. Gary D. Hammer, M.D., Ph.D. is the director of the Endocrine Oncology Program of the Comprehensive Cancer Center at the University of Michigan that specializes in the multidisciplinary care of patients with various cancer genetic syndromes and endocrine neoplasias including thyroid cancer, adrenocortical carcinoma, and neuroendocrine tumors. Dr. Hammer oversees an active research program with an emphasis on dysregulated growth of adrenocortical stem/progenitor cells in development and cancer. Donald McDonnell, Ph.D. is the chairman of the Department of Pharmacology and Cancer Biology at Duke University School of Medicine and co-director of the Women's Cancer Program within the Duke Cancer Institute. His research focuses on the genetic and pharmacological dissection of the nuclear receptor signal transduction pathways. In addition, the Editorial Board has been expanded to encompass experts not only in the various types of cancers, but in newer technologies including both DNA/RNA-based technologies and metabolomics. This year, we have increased the number of experts in endocrine neoplasias and in clinical studies to reflect the diversity of topics covered. The topic of hormones and cancer is evolving, and hormone effects are being detected in many tumors not classically considered to be hormone sensitive. With the realization that disease susceptibility and responses to therapy are often gender specific and the NIH mandate to consider these differences, there will be an increased emphasis on the effects of hormones in cancer.

Changes also have been made both to the Springer Hormones and Cancer web site, http://www.springer.com/ medicine/oncology/journal/12672, and to the Endocrine Society web site, http://press.endocrine.org/hoca, to more clearly describe the journal and its contents. The board member list can be found at http://www.springer.com/ medicine/oncology/journal/12672? detailsPage= editorialBoard. We plan to add features to make the significance of the articles more broadly accessible to a wide range of readers. One such change will be to increase the number of commentaries on published articles. We welcome suggestions for added content/features from both authors and readers. Beginning with this year's issues, the cover art will be derived from one of the articles in the issue and/or will be related to a topic featured in the issue. The February issue contained reviews related to thyroid cancer and the cover featured a slide provided by Dr. Tom Giordano showing a metastatic papillary thyroid carcinoma. This issue is the first to feature a cover figure from a research article. We seek reviews informing readers of major advances in all areas covered by the journal. These may be perspectives or longer and more comprehensive reviews. We also plan to continue to have series of thematic reviews of timely topics. This is an exciting time for the journal, and I look forward to serving as editor-inchief. 